
cancers; treatment

\title{
Increased risk of second malignancies in chronic lymphocytic leukaemia patients as compared with follicular lymphoma patients: a Canadian population-based study
}

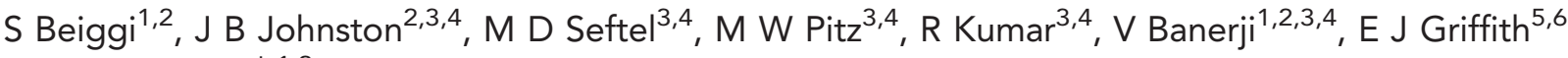 \\ and S B Gibson *,1,2
}

${ }^{1}$ Department of Biochemistry and Medical Genetics, University of Manitoba, 745 Bannatyne Avenue, Winnipeg, MB, Canada R3E OV9; ${ }^{2}$ Manitoba Institute of Cell Biology, CancerCare Manitoba, 675 McDermot Avenue, Winnipeg, MB, Canada R3E OV9; ${ }^{3}$ Department of Hematology and Medical Oncology, CancerCare Manitoba, 675 McDermot Avenue, Winnipeg, MB, Canada R3E OV9; ${ }^{4}$ Department of Internal Medicine, University of Manitoba, 820 Sherbrook Street, Winnipeg, MB, Canada R3E 2N2; ${ }^{5}$ Department of Community Health Sciences, University of Manitoba, 750 Bannatyne Avenue, Winnipeg, MB, Canada R3E OW3 and ${ }^{6}$ Department of Epidemiology and Cancer Registry, CancerCare Manitoba, 675 McDermot Avenue, Winnipeg, MB, Canada R3E OV9

Background: Chronic lymphocytic leukaemia (CLL) patients have an increased risk of other malignancies. This may be due to surveillance bias, treatment or immunosuppression.

Methods: Cohort study of 612 consecutively diagnosed CLL patients in a Canadian province, with comparisons to follicular lymphoma (FL) patients.

Results: Treated CLL patients had a 1.7-fold increased risk of second cancers compared with untreated CLL patients. As compared with untreated FL patients, untreated CLL patients had a two-fold increased incidence of second malignancies.

Conclusion: Chronic lymphocytic leukaemia patients have an inherent predisposition to second cancers and the incidence is further increased by treatment.

Chronic lymphocytic leukaemia/small lymphocytic lymphoma (CLL/SLL) is the most common leukaemia in adults (Johnston et al, 2009). Primary causes of death consist of second cancers, progressive disease and infections (Wierda et al, 2009; Yoon et al, 2012). Patients with CLL/SLL have an impaired immune system, and this may partly explain the increased incidence of second malignancies (Molica, 2005). However, the increased incidence of second cancers may also be related to an inherent predisposition to malignancy, to the effects of systemic therapy or to surveillance bias due to close lifetime monitoring.
A retrospective population study in Manitoba, Canada, 40 years ago showed that the risk of all cancers was increased three-fold in CLL, while the increase in skin cancers was eight-fold (Manusow and Weinerman, 1975). Subsequent studies have shown the same pattern (Tsimberidou et al, 2009; Royle et al, 2011b). In these studies, immunophenotyping for CLL/SLL diagnosis was not utilised. Therefore, at least $10 \%$ of patients may have been misclassified (Seftel et al, 2009). Second, most Cancer Registries do not capture non-melanoma skin cancers (NMSCs), the major contributor to the incidence of second cancers in CLL/SLL patients. 
Furthermore, a cancer-free control population is commonly utilised that may lead to inaccuracy, as one cancer diagnosis increases the risk of development and detection of subsequent malignancies (Nugent et al, 2005; Morton et al, 2010; Royle et al, 2011b).

In the current study, we used Cancer Registry and immunophenotypic data in order to create a population-based cohort of confirmed CLL/SLL patients. Importantly, this cancer registry routinely captures NMSCs, as NMSCs are reportable in Manitoba. We compared CLL/SLL patients to the general population of Manitoba as well as to patients with follicular lymphoma (FL), another indolent B-cell malignancy with prognosis and long-term clinical surveillance similar to CLL/SLL. Furthermore, in Manitoba, FL patients received similar chemotherapy regimens to CLL/SLL patients (Chlorambucil and Fludarabine) for the majority of this study period. Comparison with a similar cancer cohort significantly reduces biases (e.g., surveillance and treatment bias) that could be introduced by comparing a cancer cohort with the cancer-free population. We also evaluated the impact of systemic therapy on the incidence of second cancers. Finally, we examined malignancies in CLL/SLL patients both before and after the diagnosis of CLL/SLL.

\section{METHODS}

The CLL/SLL cohort (subsequently referred to as CLL) has been previously published (Seftel et al, 2009). All immunophenotypically confirmed CLL and FL diagnoses in Manitoba between January 1998 and December 2003 were obtained from the Manitoba Cancer Registry. In accordance with the previous study (Seftel et al, 2009), NCI-WG 1996 diagnosis criteria (Cheson et al, 1996) were used. Ethics approval was obtained from the University of Manitoba Health Research Ethics Board.

We used a retrospective cohort design. Standardised incidence ratios (SIRs) were calculated as the ratio of observed number of second cancers in CLL patients to an expected number derived from age- and gender-standardised incidence of first cancers in the general population or second malignancies in FL patients. Person-years at risk was calculated for each person in the CLL or FL cohort from the date of diagnosis of CLL or FL, up to the date of diagnosis of the second cancer, date of death or 31 December 2009, whichever came first. Person-years at risk for Manitoba population was estimated by the sum of the Manitoba population for each year of the study (1998-2009). Three patients (0.6\%) were diagnosed with a second cancer within 30 days of their CLL diagnosis and they remained in the study. Patients with a history of previous cancer and patients diagnosed with CLL at the time of death $(n=11)$ were excluded from SIR calculations. Sub-distribution hazard ratios were calculated using a competing risk regression model considering age and gender as covariates and death before a second cancer a competing risk (Fine and Gray, 1999). For purposes of the analysis, if patients were initiated on systemic treatment less than 6 months prior to the diagnosis of a second cancer they were considered untreated. Odds ratios (ORs) for history of previous cancers were estimated using a logistic regression model, adjusting for age, gender and year of diagnosis. Prevalence of previous cancers in the CLL cohort was compared with the prevalence of first cancers in the Manitoba population for the same age, gender and calendar year groups.

Data management and analysis were performed using SAS 9.2 (SAS Institute Inc., Cary, NC, USA) and Stata 11.2 (Stata Corp., College Station, TX, USA).

\section{RESULTS}

Between 1998 and 2003, 612 CLL and 372 FL patients were diagnosed in Manitoba. For CLL and FL cohorts, median age at diagnosis was 71 (31-101) years and 63 (24-92) years, male-tofemale ratios were $1.3: 1$ and $1.2: 1$, median follow-up was 6.4 $(0-12)$ years and $6.9(0-12)$ years, and median time to development of a second cancer was $3.3(0-11.4)$ and $4.0(0-11.3)$ years, respectively (Supplementary Table $\mathrm{S} 1$ ).

Chronic lymphocytic leukaemia patients had a 1.8 -fold increase in the relative risk of a second cancer (SIR 1.79, 95\% CI 1.30-2.45) compared with age- and gender-standardised FL patients. This increased risk was evident for all ages and both genders. The risk of NMSCs alone was more than two-fold higher in CLL patients compared with FL patients (SIR 2.27, 95\% CI 1.38-3.74) and when stratified by gender this was only significant in males (SIR 3.12, 95\% CI 1.60-6.10). Chronic lymphocytic leukaemia patients also

Table 1. Increased rates of second malignancies in patients with CLL as compared with FL patients and the general population of Manitoba

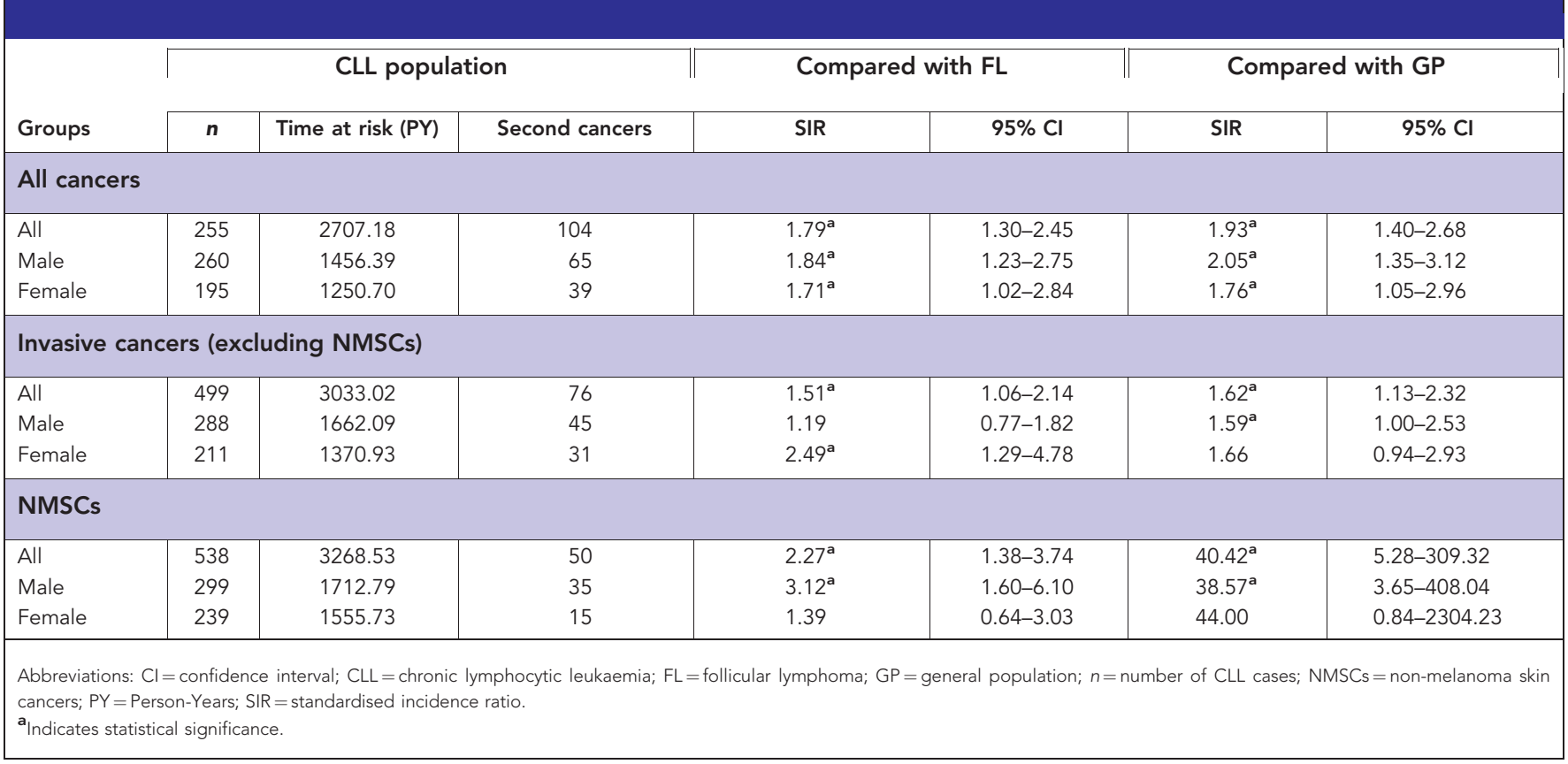




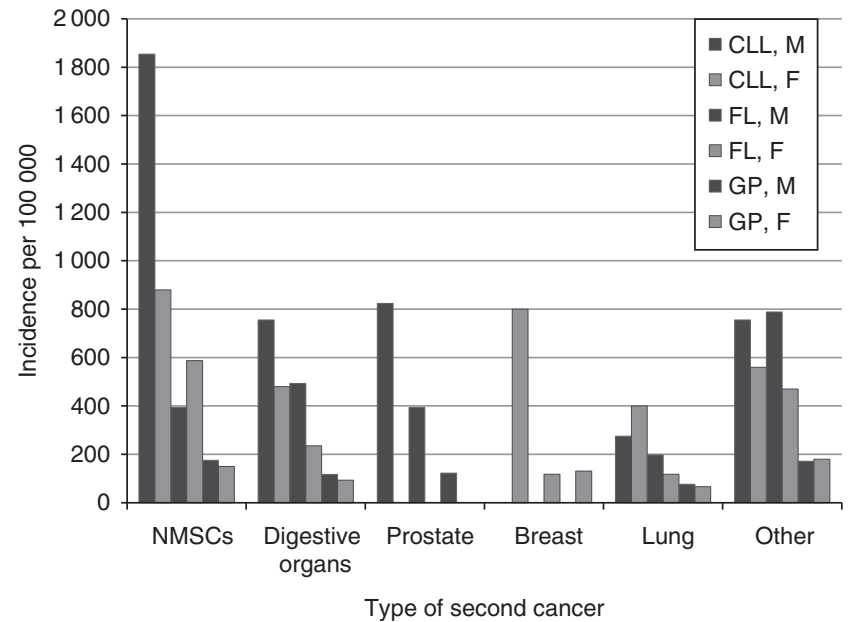

Figure 1. Site-specific incidence rates of second malignancies in CLL patients as compared with FL patients and the general population of Manitoba, by gender. Unadjusted incidence rates are calculated from 1998 to 2009 and are expressed per 100000 persons. CLL = chronic lymphocytic leukaemia; $F=$ female; $F L=$ follicular lymphoma;

$\mathrm{GP}=$ general population; $\mathrm{M}=$ male.

showed an increased risk of second cancers when compared with the general population of Manitoba (SIR 1.93, 95\% CI 1.40-2.68; Table 1, Supplementary Tables S2 and S3).

The most common second cancer among CLL patients was NMSCs (37\%), followed by cancers of digestive organs (16\%), prostate $(12 \%)$, breast $(10 \%)$ and lung $(9 \%)$. Second cancers in FL and the general population followed the same general pattern (Figure 1, Supplementary Tables S4-S6).

Both treated CLL and FL patients had an increased risk of second cancers compared with untreated CLL and FL patients, respectively. Untreated CLL patients had a two-fold increased incidence of second malignancies compared with untreated FL patients (Table 2, Supplementary Figures S1 and S2).

When previous cancers were investigated, patients with previous cancers showed a higher chance of developing CLL (OR 1.43, 95\% CI 1.03-1.97, $P=0.0322$ ). However when adjusted for age, the association disappeared (OR 0.99, 95\% CI 0.70-1.41, $P=0.9712$; OR age $1.58,95 \%$ CI $1.42-1.76, P<0.0001)$. Gender and year of diagnosis did not significantly improve the model. In addition, no significant difference in prevalence of previous cancers was observed between the CLL patients at the time of CLL diagnosis and the general Manitoba population of the same age and gender (data not shown).

\section{DISCUSSION}

In this population-based cohort study, patients with CLL had an almost two-fold increased incidence of second malignancies compared with patients with FL. Non-melanoma skin cancers were the most common second malignancy (37\%), with a 2.3 -fold increased risk in CLL compared with FL. When stratified by gender, this risk was only significant in male patients, and may be explained by the fact that men are more likely to work outdoors and receive greater exposure to UV radiation (Chen et al, 2013). The elevated incidence of NMSCs is consistent with previous reports, where skin cancers constituted $29.9 \%$ of all second cancers (Tsimberidou et al, 2009) and patients with CLL had a 3.66-fold increased risk of NMSCs when compared with the general population (Schöllkopf et al, 2007).

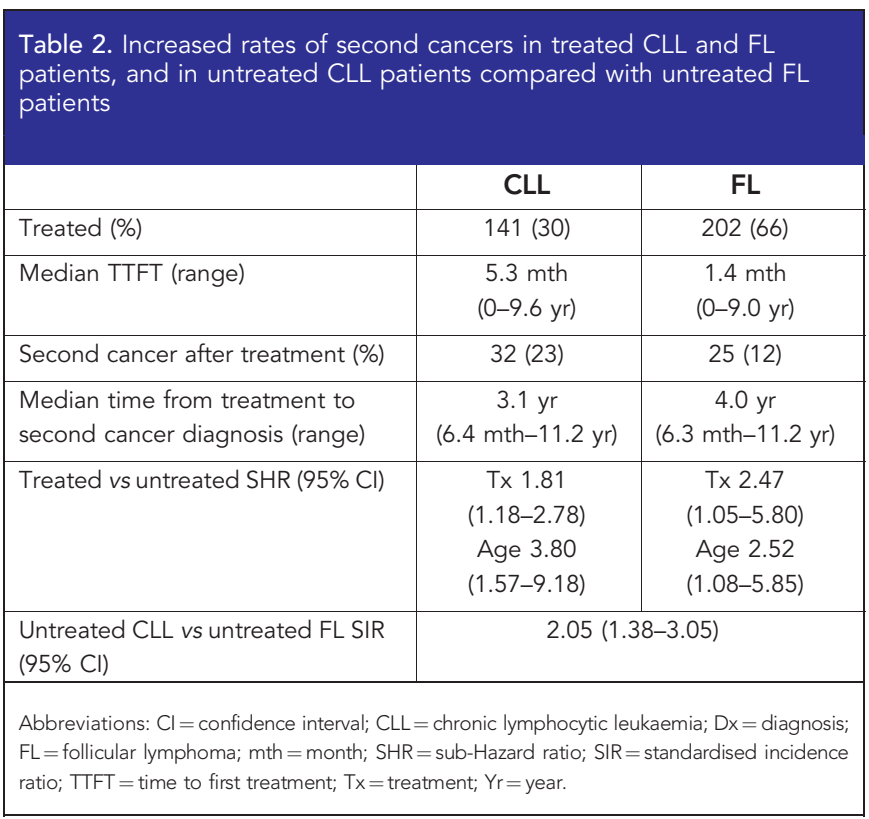

The increased incidence of second malignancies in CLL has been partly attributed to the profound immune deficiency seen in this disease (Tsai et al, 2009). An increased incidence of NMSCs has been closely related to immunosuppression after renal transplantation and other immunosuppressed individuals (Greene et al, 1978; García et al, 2013). Other studies have documented a high incidence of skin cancers in CLL patients and have noted a more aggressive disease course and increased mortality when compared with patients without CLL (Royle et al, 2011a). In our CLL cohort, deeper and longer degrees of immunosuppression might explain the high incidence of skin cancers in these patients as compared with the FL patients. Furthermore, it has been shown that patients with breast and colorectal cancers and a pre-existent CLL have an inferior overall and cancer-specific survival. This is suggested to be due to a less-effective immune response in CLL patients that consequently provides a more permissive environment for metastases (Solomon et al, 2013).

Chemotherapy can increase the incidence of second malignancies in CLL patients (Molica, 2005). In our study, the treated CLL and FL patients had substantially increased rates of second malignancies compared with untreated CLL and FL patients, respectively. However, it is important to highlight the two-fold higher incidence in untreated CLL patients compared with untreated FL patients, suggesting that treatment is not the sole contributor to the elevated risk of second cancers observed in CLL patients.

We have previously observed an increased incidence of nonHodgkin lymphoma, melanoma, lung, prostate and breast cancers following a diagnosis of NMSC (Nugent et al, 2005). This suggests a common factor in the aetiology of these malignancies. In addition, an increased incidence of CLL has been observed in families of patients with colon cancer (Teruya-Feldstein et al, 2002) and an increased incidence of breast cancer has been noted in first-degree relatives of CLL patients (Linet et al, 1989). However, the role of genetic factors for secondary cancers in CLL patients is mitigated by our observation that the incidence of other malignancies is increased only after the diagnosis of CLL, rather than beforehand. Finally, the increased rates of second cancers in CLL patients cannot be attributed to treatment or surveillance bias, as in Manitoba FL patients undergo routine followup in a similar fashion to CLL patients.

It should be noted that CLL patients were diagnosed using the diagnostic criteria from 1996, which required patients to have a peripheral blood lymphocyte count of $>5 \times 10^{9}$ per liter (Cheson et al, 1996). The updated definition from 2008 requires a 
peripheral blood B-cell count of $>5 \times 10^{9}$ per liter (Hallek et al, 2008). Thus, a small proportion of our patients may have had monoclonal B-cell lymphocytosis. Second, patients diagnosed between 1998 and 2003 would have received treatment with single-agent chemotherapy. It remains to be seen if the second cancer rate will increase in patients receiving chemoimmunotherapy. Finally, our relatively small cohort and short follow-up yielded few second malignancies.

In conclusion, we have observed that the risk of second cancers in CLL patients is not only greater than the general population, but is also greater than a closely related lymphoproliferative disorder, FL. This increased risk is independent of treatment or surveillance bias. Of the second malignancies, skin cancers were the most common type. Heightened awareness about risks of second cancers should be communicated to improve the care and outcome of CLL patients.

\section{ACKNOWLEDGEMENTS}

This work was supported by a CancerCare Manitoba Foundation grant and a Manitoba Health Research Council studentship. The funding source had no part in study design, in the collection, analysis and interpretation of data, in the writing of the manuscript and in the decision to submit the manuscript for publication. We are grateful to Pascal Lambert and Dr Zoann Nugent for statistical consultation.

\section{CONFLICT OF INTEREST}

James Johnston is on the advisory board of Roche and received research funding from Roche Pharmaceuticals. Rajat Kumar is on the advisory board of Celgene. Other authors have no disclaimers.

\section{REFERENCES}

Chen AC, Halliday GM, Damian DL (2013) Non-melanoma skin cancer: carcinogenesis and chemoprevention. Pathology 45(3): 331-341.

Cheson BD, Bennett JM, Grever M, Kay N, Keating MJ, O’Brien S, Rai KR (1996) National Cancer Institute-sponsored Working Group guidelines for chronic lymphocytic leukemia: revised guidelines for diagnosis and treatment. Blood 87: 4990-4997.

Fine JP, Gray RJ (1999) A proportional hazards model for the subdistribution of a competing risk. J Am Stat Assoc 94: 496-509.

García JB, Suárez-Varela MM, Vilata JJ, Marquina A, Pallardó L, Crespo J (2013) Risk factors for non-melanoma skin cancer in kidney transplant patients in a Spanish population in the Mediterranean region. Acta Derm Venereol 93: 422-427.

Greene MH, Hoover RN, Fraumeni Jr JF (1978) Subsequent cancer in patients with chronic lymphocytic leukemia-a possible immunologic mechanism. J Natl Cancer Inst 61: 337-340.

Hallek M, Cheson BD, Catovsky D, Caligaris-Cappio F, Dighiero G, Döhner H, Hillmen P, Keating MJ, Montserrat E, Rai KR, Kipps TJ (2008) Guidelines for the diagnosis and treatment of chronic lymphocytic leukemia: a report from the International Workshop on Chronic Lymphocytic Leukemia updating the National Cancer Institute-Working Group 1996 guidelines. Blood 111: 5446-5456.
Johnston JB, Gibson SB, Seftel MD (2009) Chronic lymphocytic leukemia. In: Greer JP, Foerster J, Lukens J, Paraskevas F, Rodgers GM, Glader B (eds) Wintrobe's Clinical Hematology. Lippincott Williams \& Wilkins: Baltimore, pp 2256-2275.

Linet MS, Van Natta ML, Brookmeyer R, Khoury MJ, McCaffrey LD, Humphrey RL, Szklo M (1989) Familial cancer history and chronic lymphocytic leukemia. A case-control study. Am J Epidemiol 130: 655-664.

Manusow D, Weinerman BH (1975) Subsequent neoplasia in chronic lymphocytic leukemia. JAMA 232: 267-269.

Molica S (2005) Second neoplasms in chronic lymphocytic leukemia: incidence and pathogenesis with emphasis on the role of different therapies. Leuk Lymphoma 46: 49-54.

Morton LM, Curtis RE, Linet MS, Bluhm EC, Tucker MA, Caporaso N, Ries LAG, Fraumeni Jr JF (2010) Second malignancy risks after non-Hodgkin's lymphoma and chronic lymphocytic leukemia: differences by lymphoma subtype. J Clin Oncol 28: 4935-4944.

Nugent Z, Demers AA, Wiseman MC, Mihalcioiu C, Kliewer EV (2005) Risk of second primary cancer and death following a diagnosis of nonmelanoma skin cancer. Cancer Epidemiol Biomarkers Prev 14: 2584-2590.

Royle JA, Baade PD, Joske D, Girschik J, Fritschi L (2011a) Second cancer incidence and cancer mortality among chronic lymphocytic leukaemia patients: a population-based study. Br J Cancer 105: 1076-1081.

Royle JS, Baade P, Joske D, Fritschi L (2011b) Risk of second cancer after lymphohematopoietic neoplasm. Int J Cancer 129: 910-919.

Schöllkopf C, Rosendahl D, Rostgaard K, Pipper C, Hjalgrim H (2007) Risk of second cancer after chronic lymphocytic leukemia. Int J Cancer 121: 151-156.

Seftel MD, Demers AA, Banerji V, Gibson SB, Morales C, Musto G, Pitz MW, Johnston JB (2009) High incidence of chronic lymphocytic leukemia (CLL) diagnosed by immunophenotyping: a population-based Canadian cohort. Leuk Res 33: 1463-1468.

Solomon BM, Rabe KG, Slager SL, Brewer JD, Cerhan JR, Shanafelt TD (2013) Overall and cancer-specific survival of patients with breast, colon, kidney, and lung cancers with and without chronic lymphocytic leukemia: a SEER population-based study. J Clin Oncol 31: 930-937.

Teruya-Feldstein J, Greene J, Cohen L, Popplewell L, Ellis NA, Offit K (2002) Analysis of mismatch repair defects in the familial occurrence of lymphoma and colorectal cancer. Leuk Lymphoma 43: 1619-1626.

Tsai H-T, Caporaso NE, Kyle RA, Katzmann JA, Dispenzieri A, Hayes RB, Marti GE, Albitar M, Ghia P, Rajkumar SV, Landgren O (2009) Evidence of serum immunoglobulin abnormalities up to 9.8 years before diagnosis of chronic lymphocytic leukemia: a prospective study. Blood 114: 4928-4932.

Tsimberidou A-M, Wen S, McLaughlin P, O'Brien S, Wierda WG, Lerner S, Strom S, Freireich EJ, Medeiros LJ, Kantarjian HM, Keating MJ (2009) Other malignancies in chronic lymphocytic leukemia/small lymphocytic lymphoma. J Clin Oncol 27: 904-910.

Wierda WG, O'Brien S, Wang X, Faderl S, Ferrajoli A, Do K-A, GarciaManero G, Cortes J, Thomas D, Koller C, Burger J, Lerner S, Kantarjian H, Keating M (2009) Characteristics associated with important clinical end points in patients with chronic lymphocytic leukemia at initial treatment. J Clin Oncol 27: 1637-1643.

Yoon J-Y, Lafarge S, Dawe D, Lakhi S, Kumar R, Morales C, Marshall A, Gibson SB, Johnston JB (2012) Association of interleukin-6 and interleukin-8 with poor prognosis in elderly chronic lymphocytic leukemia patients. Leuk Lymphoma 53(9): 1735-1742.

Supplementary Information accompanies this paper on British Journal of Cancer website (http://www.nature.com/bjc) 